\title{
LEFT VENTRICULAR PSEUDOANEURYSM IN BEHÇET’S DISEASE: A CASE REPORT
}

\begin{abstract}
Beatriz Mota Tiburcio ${ }^{1, \star}$, Eduardo José do Rosario e Souza ${ }^{1}$, Marcella Maria Soares Melo ${ }^{1}$, Carolina Lais Ladeira Estefani ${ }^{1}$, Evelyn Joyce Corgosinho ${ }^{1}$, Marcelo Rocha Scaramussa ${ }^{1}$, Italo Magalhaes Gusmao ${ }^{1}$, Larissa Andrade Lourenço ${ }^{1}$
\end{abstract}

1.Santa Casa de Misericórdia de Belo Horizonte, Belo Horizonte (MG), Brazil.

*Corresponding author: biamotatiburcio@gmail.com

\section{BACKGROUND}

Behçet's disease is a multisystemic vasculitis that mainly affects young adults, characterized by findings of recurrent oral and/or genital ulcers, uveitis and skin lesions. However, other manifestations are described, such as the involvement of the central nervous system and also of the great vessels of the body. Left ventricular pseudoaneurysm is a rare manifestation of the disease.

\section{CASE REPORT}

A15-year-old male, previously healthy, presented with left chest pain, fatigue, progressive dyspnea, weight loss, adynamia, daily evening fever and chills. Symptoms persisted for a few months and, associated with it, the patient reported recurrent oral ulcers. On admission, he presented oral and genital ulcers, generalized lymphadenomegaly, hepatosplenomegaly and bilaterally reduced pedal pulses. Laboratory presented with hypochromic and microcytic anemia, high inflammatory tests, negative viral and bacterial serology, ANA 1:160 fine nuclear. Biopsy of the lymph node showed reactive infiltrate. A transthoracic echocardiogram was performed, showing a pseudoaneurysm at the tip of the left ventricle, with negative blood cultures. He was submitted to a surgical approach, uneventful. In a postoperative follow-up examination, he presented with ventricular and aortic pseudoaneurysm. Rheumatology team evaluates the patient and establishes the diagnosis of Behçet's disease with vascular and cardiac involvement. Systemic corticosteroid therapy and immunosuppression with cyclophosphamide were started. Submitted to a new surgical approach for the correction of pseudoaneurysms. Induction was maintained with monthly pulse therapy with cyclophosphamide for 6 months and subsequent maintenance with azathioprine. There was no recurrence or appearance of pseudoaneurysms.

\section{CONCLUSION}

Involvement of large vessels is an uncommon form of manifestation of Behçet's disease, only $3 \%$ to $5 \%$ of patients. The formation of pseudoaneurysms is an even rarer finding, and it confers high morbidity and mortality on the patient if not properly managed.

\section{KEYWORDS}

Behçet, Pseudoaneurysm, Large vessels, Vasculitis. 